\title{
Dual effect of Plasmodium-infected erythrocytes on dendritic cell maturation
}

\author{
Esther Bettiol ${ }^{1 \dagger}$, Daniel Carapau ${ }^{1 \dagger}$, Cristina Galan-Rodriguez ${ }^{1}$, Carlos Ocaña-Morgner ${ }^{1,2}$, Ana Rodriguez $^{1 *}$
}

\begin{abstract}
Background: Infection with Plasmodium is the cause of malaria, a disease characterized by a high inflammatory response in the blood. Dendritic cells (DC) participate in both adaptive and innate immune responses, influencing the generation of inflammatory responses. DC can be activated through different receptors, which recognize specific molecules in microbes and induce the maturation of DC.
\end{abstract}

Methods: Using Plasmodium yoelii, a rodent malaria model, the effect of Plasmodium-infected erythrocytes on DC maturation and TLR responses have been analysed.

Results: It was found that intact erythrocytes infected with P. yoelii do not induce maturation of DC unless they are lysed, suggesting that accessibility of parasite inflammatory molecules to their receptors is a key issue in the activation of DC by P. yoelii. This activation is independent of MyD88. It was also observed that pre-incubation of DC with intact $P$. yoelii-infected erythrocytes inhibits the maturation response of DC to other TLR stimuli. The inhibition of maturation of DC is reversible, parasite-specific and increases with the stage of parasite development, with complete inhibition induced by schizonts (mature infected erythrocytes). Plasmodium yoelii-infected erythrocytes induce a broad inhibitory effect rendering DC non-responsive to ligands for TLR2, TLR3, TLR4, TLR5, TLR7 and TLR9.

Conclusions: Despite the presence of inflammatory molecules within Plasmodium-infected erythrocytes, which are probably responsible for DC maturation induced by lysates, intact Plasmodium-infected erythrocytes induce a general inhibition of TLR responsiveness in DC. The observed effect on DC could play an important role in the pathology and suboptimal immune response observed during the disease. These results help to explain why immune functions are altered during malaria, and provide a system for the identification of a parasite-derived broad inhibitor of TLR-mediated signaling pathways.

\section{Background}

Malaria is one of the most lethal infectious diseases worldwide. Acquired immunity to malaria is directed to the blood stage of the parasite and is slow to develop, taking more than five years to develop in individuals living in endemic areas [1]. A better understanding of innate and acquired immunity to Plasmodium parasites is required for the development of the development of effective anti-malaria strategies.

Dendritic cells (DC) play a major role in host responses to pathogens, influencing both innate and adaptive immunity, as it is evident by their unique

\footnotetext{
* Correspondence: Ana.Rodriguez@nyumc.org

+ Contributed equally

'Department of Medical Parasitology, New York University School of Medicine, 341 East 25th street, New York, NY 10010, USA
}

capacity to activate naïve $\mathrm{T}$ cells and polarize $\mathrm{CD} 4^{+}$ $\mathrm{T}$ cell responses. Activation of Toll-like receptors (TLR) induces the maturation of $\mathrm{DC}$, which is required for the generation of effective innate and $\mathrm{T}$ cell-mediated responses [2]. DC maturation is associated with the expression at the cell surface of MHC-peptide complexes for antigen presentation to $\mathrm{T}$ cells (signal 1 ), costimulatory molecules such as CD40, CD80/B7.1 and CD86/B7.2 (signal 2), as well as secretion of cytokines such as IL-12, that induce $\mathrm{T}$ cell polarization [3]. DC maturation can also be induced by activation of the inflammasome [4], which can be triggered by different molecules, such as alum or uric acid [5].

Whether DC mature normally during malaria has been a mater of debate [6]. Studies using DC, either differentiated in vitro or directly isolated from infected

\section{() Biomed Central}

(c) 2010 Bettiol et al; licensee BioMed Central Ltd. This is an Open Access article distributed under the terms of the Creative Commons Attribution License (http://creativecommons.org/licenses/by/2.0), which permits unrestricted use, distribution, and reproduction in any medium, provided the original work is properly cited. 
mice, have shown an inhibition of DC maturation by blood stages of human-infective (Plasmodium falciparum) and rodent-infective Plasmodium species [7-11]; however, other studies found effective DC maturation, again in both rodent and human models [12-14]. Analysis of malaria infections in mice also produced controversial results showing either activation $[15,16]$ or inhibition of DC function [17,18]. Finally, analysis of DC during human malaria infections has shown that the frequency of BDCA3+ myeloid DCs was significantly increased during acute disease [19], suggesting that the distribution of DC subpopulations is affected during human malaria. Recent studies in mice have contributed to the clarification of these apparent contradictions by demonstrating that the effect of Plasmodium on DC is dependent on diverse factors, including the parasite strain [20], the subpopulation of DC studied, the time after infection, and the size of the inoculum [21].

In recent years, extensive characterization of TLR receptors (TLR1 to 12) has been performed, providing new insights on the specificity of innate immunity [22]. Among eukaryotic parasites, diverse TLR ligands have been identified, such as glycophosphatidyl-inositol (GPI) from Trypanosoma cruzi [23], lipophosphoglycan from Leishmania [24] or profilin from Toxoplasma [25]. Two different Plasmodium-derived molecules have been identified as activators of antigen-presenting cells through TLR-dependent mechanisms. Plasmodium-derived GPI [26] and DNA-covered haemozoin [27,28] activate TLR2 and TLR9, respectively. While GPI induces secretion of TNF by macrophages [26], haemozoin induces maturation of DC and secretion of TNF, IL-12 and IL-6 [27]. In these studies, activation of TLR has been performed using molecules purified from parasites, or else synthetic analogues, but TLR stimulation using whole parasites or lysates was not evaluated.

In this work, the role of Plasmodium yoelii-infected erythrocytes in the maturation of DCs was characterized. It was also addressed whether the effects of phagocytosing high amounts of erythrocytes per se was associated with modulation of DC maturation. By assessing the level of expression of co-stimulatory molecules, it is shown that whole $P$. yoelii-infected erythrocytes do not induce a strong maturation response in bone marrow-derived DC in vitro. This lack of response was not dependent on parasite stage, viability or time of incubation. Conversely, lysates of infected erythrocytes induced a MyD88-independent activatory response in DC. The inhibition of DC maturation by infected erythrocytes was parasite-specific, since increasing phagocytosis of uninfected erythrocytes by DC did not have the same effect. It was also observed that pre-incubation of DC with infected erythrocytes inhibits the DC maturation response triggered by different TLR ligands, as measured both by the expression of co-stimulatory molecules and the secretion of bioactive IL- 12 .

\section{Methods}

\section{Animals and parasites}

Balb/C, Swiss Webster and C57BL/6 mice were purchased from Taconic and housed in NYU animal facility according to protocols approved by IACUC. MyD88deficient C57BL/6 mice were kindly provided by Dr. Elizabeth Nardin (New York University). Plasmodium yoelii 17XNL parasites were obtained from Swiss Webster mice infected intraperitoneally.

\section{Culture of mouse bone marrow-derived myeloid DC}

DC were generated from bone marrow precursors as previously described [29]. Briefly, bone marrow cells from the femur and tibia of BALB/c mice of 8-10 weeks of age were harvested and cultured for 8 to 10 days in $80 \%$ complete DMEM (containing 10\% FBS, penicillin $100 \mathrm{U} / \mathrm{ml}$, streptomycin $0.1 \mathrm{mg} / \mathrm{ml}$, glutamine $0.292 \mathrm{mg} / \mathrm{ml}$ ), and 20\% of GM-CSF (Granulocyte/Macrophage colony-stimulating factor) conditioned medium (medium from cultures of AG8653 myeloma cells that are transfected with GM-CSF). DC culture reproducibly yielded more than $85 \%$ of CD11c+ cells. DC from BALB/c, Swiss-Webster or C57BL/6 mice were used in this study and similar results were observed with DC from both mouse strains. All experiments were performed with DC derived from Swiss Webster mice, except for the results shown in Figure 1A, where the staining of MHC-I and $\mathrm{MHC}-\mathrm{II}$ required the use of $\mathrm{DC}$ from $\mathrm{BALB} / \mathrm{c}$ mice and Figure 1B, where MyD88-deficient DC can only be obtained from C57BL/6 mice. At least one of the confirmatory experiments for all results obtained with SwissWebster DC, was performed using BABL/c-derived DC. These results are not shown and are noted in the figure legends as "Figures are representative of one out of three (or two) independent experiments." No differences were found between the responses of DC derived from the different mice strains.

\section{Isolation of parasites and erythrocytes components}

Schizonts were isolated from the interphase of $45 \%$ Percoll gradient spun for 15 minutes at $450 \mathrm{~g}$ at RT. Preparations of over $90 \%$ schizonts were obtained. Fractions comprised of Plasmodium schizont, trophozoites or else mixed uninfected erythrocytes+ ring stages, were isolated using a multilayer Percoll gradient (65\%-45\%) as modified from Kariuki et al [30]. Briefly, P. yoelii schizonts were collected from the PBS-45\% interphase, while trophozoites were collected from the $45-65 \%$ interphase, and uninfected erythrocytes+rings from the pellet.

Plasmodium yoelii and control lysates were prepared by freeze-thawing five times schizonts or uninfected 


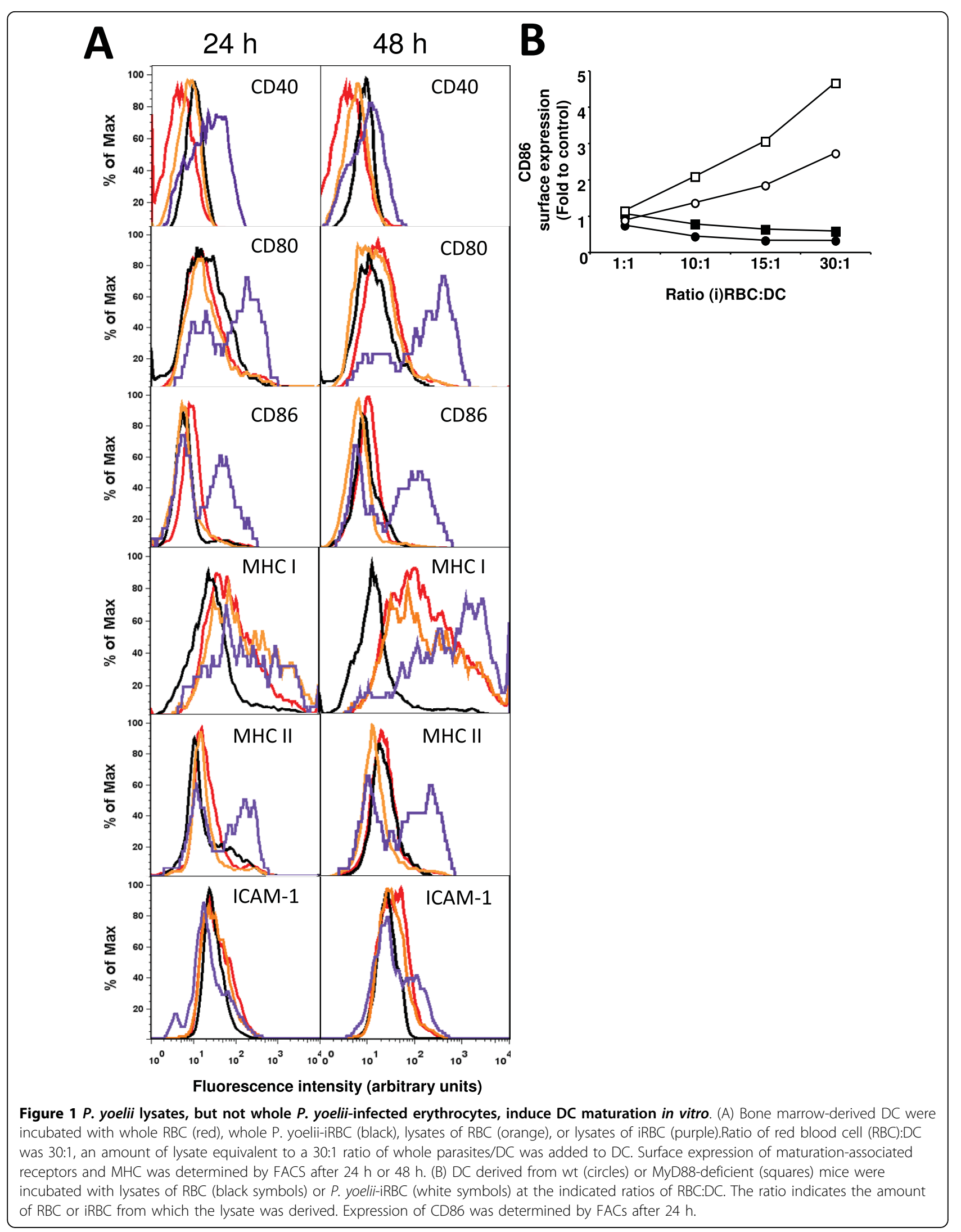


erythrocytes, respectively. An amount of lysate equivalent to a 30:1 ratio of whole parasites/DC was added to DC. Food vacuoles were isolated following the protocol described by Saliba et al [31]. Schizonts were saponized for a few seconds by resuspending them in a solution of $0.5 \mathrm{mg} / \mathrm{ml}$ saponin (Sigma-Aldrich), followed by two washes with PBS. Food vacuoles isolated from $0.3 \times 10^{9}$ to $1 \times 10^{9}$ saponized schizonts were added per $10^{6} \mathrm{DC}$. To fix parasites, schizonts were rinsed in PBS, resuspended in 4\% paraformaldehyde and incubated for 15 minutes at room temperature. Finally, schizonts were rinsed three times with PBS. To induce phosphatidyl-serine flipping, control erythrocytes were treated with $1 \mu \mathrm{M}$ ionomycin in DMSO (Sigma-Aldrich) for 30 minutes at $37 \mathrm{C}$, rinsed and plated on cells. To isolate haemoglobin, blood of uninfected Swiss Webster mice was centrifuged at $100 \mathrm{~g}$ twice to remove platelets. The blood was then passed through a Plasmodipur filter (Euro-Diagnostica, Arnhem, The Netherlands) to remove leucocytes. Erythrocytes were then lysed in water for $5 \mathrm{~min}$ on ice. PBS $10 \times$ was added to a final $1 \times$ concentration and the erythrocytes ghosts were removed by centrifugation at $16,000 \times \mathrm{g}$ for 20 minutes twice. Protein concentration was measured using Bradford dye reagent (Bio-Rad) according to the manufacturer's instructions.

Co-cultures of bone marrow-derived DC and erythrocytes After differentiation, DC were plated at $10^{6} / \mathrm{ml}$ in complete DMEM supplemented with 10\% GM-CSF conditioned medium. DC were stimulated with either P. yoellii $17 \mathrm{XNL}$-infected or uninfected erythrocytes (isolated from Swiss-Webster mice) at a 1:30 DC to erythrocytes ratio, unless indicated otherwise. For experiments where parasites were incubated for definite amounts of time, erythrocytes were removed by rinsing cells five times with PBS, while the adherent DC remain in culture.

\section{TLR ligands}

DC were stimulated with the following TLR ligands: LPS from Salmonella typhimurium (Sigma-Aldrich) for TLR4, CpG oligodeoxynucleotides (ODN 2395) for TLR9, Zymosan from Saccharomyces cerevisiae (SigmaAldrich) for TLR2, Polyinosinic-polycytidylic (PolyI:C) for TLR3, Flagellin from Salmonella typhimurium for TLR5, Pam3CysSerLys4 (P3C) for TLR1/TLR2 and loxoribine for TLR7 (all from Invivogen). Concentrations used are indicated in figure legends.

\section{Analysis of expression of co-stimulatory molecules by flow cytometry}

After harvesting, DC were incubated for $30 \mathrm{~min}$ on ice with Fc block (BD Biosciences, clone 2.4G2) at 1:500 dilution in $\mathrm{PBS}+1 \%$ Bovine Serum Albumin. DC were then stained for $1 \mathrm{~h}$ on ice in PBS $+1 \%$ Bovine Serum
Albumin with the following fluorescently labeled antibodies at 1:100 dilution: CD11c (Biolegend, clone N418 or BD Biosciences, clone HL3), CD40 (BD Biosciences, clone 3/23), and CD86 (BD Biosciences, clone GL1). Fluorescently labeled isotype controls were used in control samples and analysed in parallel to exclude nonspecific binding. To assess cell viability, 7-amino-actinomycin D (7-AAD) (BD Biosciences) was added to labeled DC 10 minutes before flow cytometry analysis. For analysis, cells were gated on expression of CD11c and absence of 7 AAD staining. Surface marker expression was assessed for CD86 as the percentage of CD86 ${ }^{\text {high }}$ cells and as mean fluorescence intensity for CD40. Annexin-FITC (Invitrogen, Molecular Probes) was used according to the manufacturer's instructions. Briefly, $10^{6}$ erythrocytes $/ \mathrm{ml}$ were labeled for $15 \mathrm{~min}$ with Annexin-FITC at 1:20 dilution and analysed by flow cytometry shortly after. All flow cytometry experiments were performed on a Becton-Dinckinson FACS Calibur and data analysed with Cell Quest or FlowJo software.

\section{Fluorescent labeling of erythrocytes}

Schizonts or erythrocytes were labeled using a protocol modified from Ing et al [16]. Briefly, uninfected or else P. yoelii-infected erythrocytes diluted in PBS at $10^{8}$ erythrocytes/ml were incubated for 15 minutes at $37 \mathrm{C}$ with $1 \mu \mathrm{M}$ CellTrace ${ }^{\mathrm{TM}}$ Far Red DDAO-SE (Molecular Probes), rinsed with PBS, incubated further for $45 \mathrm{~min}$ utes at $37 \mathrm{C}$ in complete medium and rinsed once more with PBS. For phagocytosis assays, labeled erythrocytes were centrifuged for $1 \mathrm{~min}$ at $1,000 \mathrm{rpm}$ on $\mathrm{DC}$ at a 1:30 ratio and incubated at $37 \mathrm{C}$ for $4 \mathrm{~h}$. Cells and erythrocytes were scraped on ice and directly analysed by flow cytometry. DC were gated using the size and morphology parameters (FSC, SSC) and DDAO fluorescence was analysed within the DC population.

\section{IL-12 production by DCs}

Bone marrow-derived DCs were plated as mentioned before and uninfected or $P$. yoelii-infected erythrocytes were added at different ratios of erythrocytes:DC. At different times after the start of erythrocytes incubation, LPS was added at $1 \mu \mathrm{g} / \mathrm{ml}$ for an additional $24 \mathrm{~h}$. At the end of LPS incubation, supernatants from DC/erythrocytes co-cultures were removed and spun at 13,000 $\mathrm{g}$ for 10 minutes. Supernatants were used for ELISA for quantification of IL-12p70 (BD Biosciences) according to manufacturer's instructions.

\section{Results}

Plasmodium yoelii lysates, but not whole $P$. yoelii-infected erythrocytes, induce $D C$ maturation in vitro

During malaria infection, DC and other immune cells are frequently exposed to parasite materials derived 
from lysed infected erythrocytes, but also to intact infected erythrocytes, in which case Plasmodium-derived TLR ligands will be inside infected erythrocytes. Since immature DC have high phagocytic activity they easily uptake whole $P$. yoelii-infected erythrocytes $[9,16]$. To assess the effect in DC maturation of lysed and whole intact $P$. yoelii-infected erythrocytes, DC differentiated in vitro were incubated with infected erythrocytes at the schizont stage, or else lysates corresponding to the same amount of infected erythrocytes, as well as the respective uninfected controls.

Whole infected erythrocytes did not induce increases in surface expression of any of the maturation markers, while their lysates were very active in triggering a DC response (Figure 1A). To determine whether the maturation induced by $P$. yoelii lysates is mediated through a MyD88 dependent pathway, DC derived from wild type or MyD88-deficient mice were used. It was found that $P$. yoelii lysates induce DC maturation through a MyD88 independent pathway (Figure 1B). There are three characterized inflammatory molecules in Plasmodium-infected erythrocytes: glycosylphosphatidylinositol (GPI) anchors [32], a parasite-induced polymer of degraded haem called haemozoin bound to parasite DNA [27,28] and Plasmodium-derived uric acid $[33,34]$. Since MyD88 is required for the activation mediated by GPI and DNA-covered haemozoin (TRL 2 and 9, respectively), this result suggests that the maturation induce by $P$. yoelii lysates may be mediated by uric acid, which is independent of MyD88 [33].

\section{Whole $P$. yoelii-infected erythrocytes do not induce DC maturation in vitro}

To analyse in more detail the lack of DC maturation observed with whole infected erythrocytes, surface expression of CD40 and CD86 was tested in DC incubated at different infected erythrocytes:DC ratios ranging from 1:1 to 30:1 and used TLR ligands such as LPS (TLR4 ligand) and

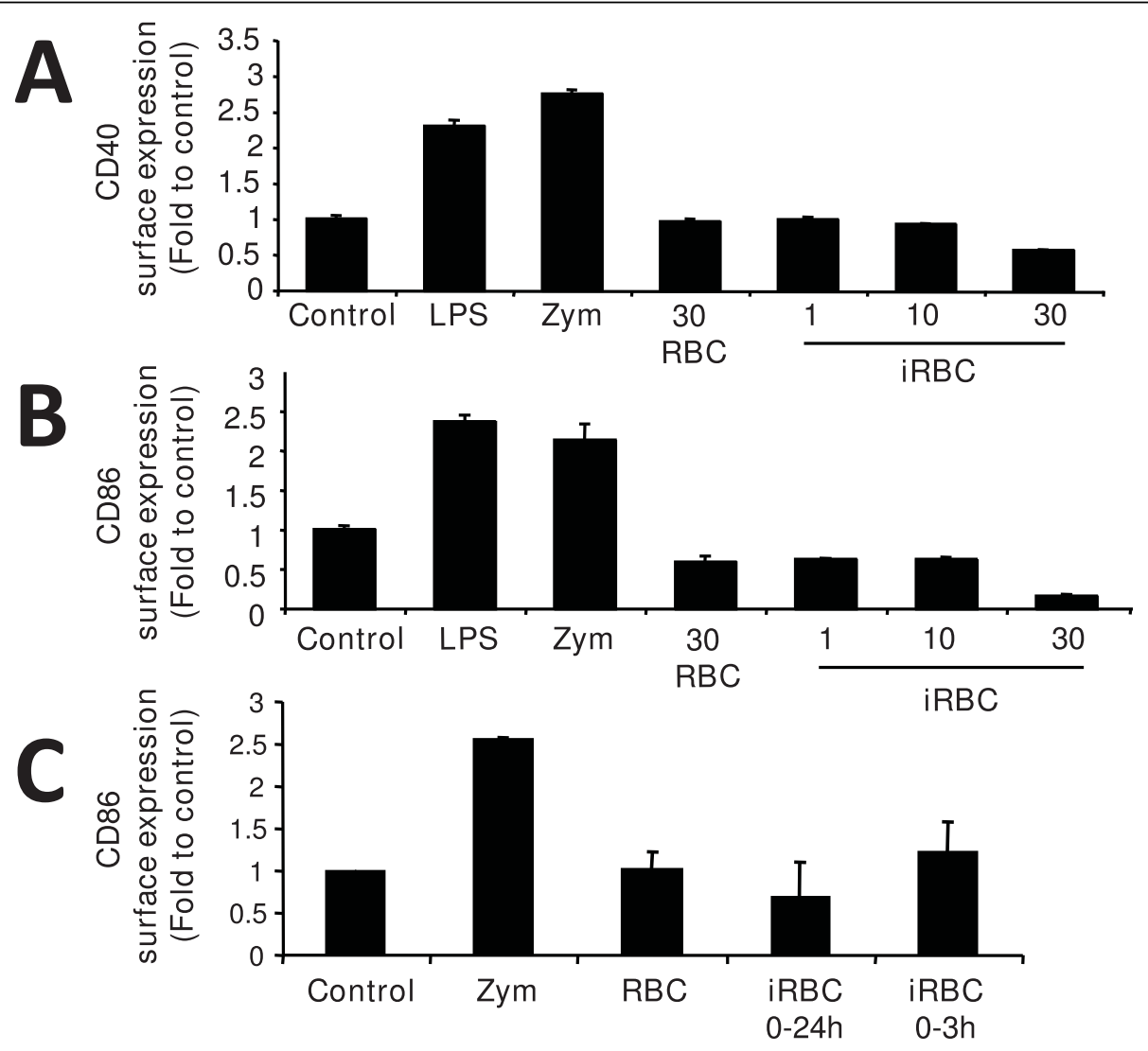

Figure 2 Whole Plasmodium-infected red blood cells (iRBC) do not induce DC maturation in vitro. Bone marrow-derived DC were incubated for $24 \mathrm{~h}$ in different conditions. Ratio of red blood cell (RBC):DC is 30:1 unless specified otherwise. Expression of CD86 and CD40 analyzed by flow cytometry is expressed as fold-to-control (DC cultured alone). LPS (Salmonella typhimurium) and Zymosan (Saccharomyces cerevisiae) were used as positive controls for DC maturation. CD40 (A) and CD86 (B) expression after incubation with RBC or P. yoelii-iRBC at different ratios. (C) Expression of CD86 at $24 \mathrm{~h}$ of culture when DC were co-incubated with iRBC for a short time ( $3 \mathrm{~h}$ pulse) and then washed, or for the whole $24 \mathrm{~h}$. Data correspond to the mean of triplicate samples \pm standard deviation. Figures are representative of one out of three independent experiments. 


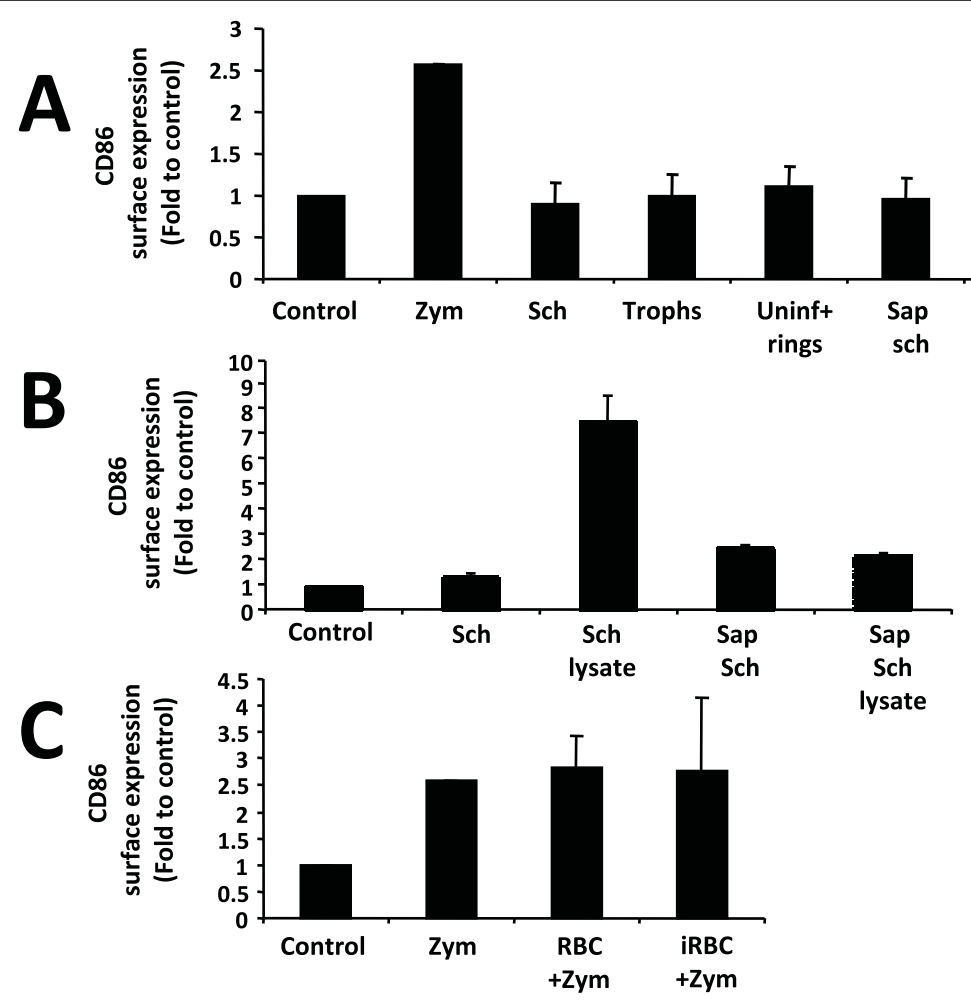

Figure $3 P$. yoelii-iRBC do not induce DC maturation in vitro. Bone marrow-derived DC were incubated for $24 \mathrm{~h}$ in different conditions. Ratio of red blood cell (RBC):DC is 30:1 (A) CD86 expression of DC co-incubated with iRBC at the schizont stage (Sch), the trophozoite stage (Trophs), a mix of uninfected RBC and rings (Uninf+rings), fixed RBC, saponized schizonts or isolated food vacuoles (FV). (B) CD86 expression of DC coincubated with iRBC at the schizont stage (Sch), its lysate, saponized schizonts (Sap sch) or its lysate. An amount of lysate equivalent to whole parasites was added to DC. (C) CD86 expression of DC co-incubated with RBC or iRBC and zymosan. Data correspond to the mean of triplicate samples \pm standard deviation.

Zymosan (TLR2 ligand) as positive controls (Figures 2A and $2 \mathrm{~B})$. Whole infected erythrocytes did not induce increases in surface expression of CD40 or CD86 in DC at infected erythrocytes:DC ratios ranging from 1:1 to 30:1 (Figures 2A and 2B). The effect of pulsing DC with infected erythrocytes for $3 \mathrm{~h}$, followed by washing and further incubation, was also analysed, showing no maturation of DC at $24 \mathrm{~h}$ of culture (Figure 1C).

As the preparations of $P$. yoelii-purified infected erythrocytes used in this study mainly contain parasites at the schizont stage, it was next investigated if other stages of intra-erythrocytic $P$. yoelii parasites were able to trigger DC maturation. To this aim, schizonts, trophozoites and rings were separated using a multilayer Percoll gradient. Rings were obtained together with uninfected erythrocytes. Each fraction was added to DC at a 30 infected erythrocytes:DC ratio for $24 \mathrm{~h}$. Maturation was not triggered by any of the parasite stages tested (Figure 3A).

To assess the role played by erythrocytes versus parasite-derived components, schizonts were treated with a mild detergent to lyse the erythrocytes membrane allowing for enrichment of isolated parasites and also purified food vacuoles. Isolated parasites did not trigger maturation of DC, neither did food vacuoles (Figure 3A). Lysates of isolated parasites also failed to induce DC maturation (Figure 3B) suggesting that the activatory molecules might be in the cytosol of the infected erythrocytes and not in the parasite itself. However, these molecules could be lost or inactivated in process of purification and lysis of the parasites. Fixed parasites, which should preserve parasite TLR ligands and prevent parasites from releasing newly synthesized metabolites also did not trigger maturation (Figure 3A). It was then tested whether an active parasite-derived inhibitory activity was causing the lack of DC maturation. DC were incubated simultaneously with infected erythrocytes and a strong activator of DC maturation, such as zymosan. It was observed that DC mature in response to zymosan regardless of the presence of infected erythrocytes, when both are added simultaneously (Figure $3 C$ ). This suggests that the absence of DC maturation in response to whole $P$. yoelii-infected erythrocytes is a result of lack of activation rather than active inhibition. 


\section{Plasmodium yoelii-infected erythrocytes inhibit DC maturation}

It was observed that $P$. yoelii-infected erythrocytes do not inhibit DC maturation when added simultaneously with a TLR ligand (Figure 3C). However, pre-incubation of DC with $P$. yoelii-infected erythrocytes inhibits DC maturation induced by LPS [9]. The effect of pre-incubation with whole $P$. yoelii-infected erythrocytes or their lysates on DC maturation was, therefore, compared. DC that had been incubated with uninfected or infected erythrocytes, or else their lysates for $24 \mathrm{~h}$ were further incubated with LPS for an additional $18 \mathrm{~h}$. The effect on expression of CD40 (Figure 4A) and CD86 (Figure 4B) was assessed by flow cytometry. This confirmed that whole $P$. yoelii-infected erythrocytes inhibit DC maturation in a dose-dependent manner, with complete inhibition at 30 infected erythrocytes:DC ratio. On the contrary, lysates at similar parasite densities did not inhibit LPS-induced maturation. Interestingly, it was found that with a 10:1 infected erythrocyte:DC ratio partial activation of DC (increased CD86 expression) occurred after $48 \mathrm{~h}$ of incubation (Figure 4B), while there was also a decrease in CD40 expression (Figure 4A). This observation, however, was not reproducible, and was found only in two out of five independent experiments. In the other three experiments, no changes in the levels of CD40 and CD86 expression were, as had been previously described [35]. These observations suggest that on certain occasions higher densities of infected erythrocytes $(30: 1)$ are required for complete inhibition, and that whole P. yoelii-infected erythrocytes can trigger a partial DC maturation phenotype, which is slow to develop (only seen at $48 \mathrm{~h}$, Figure $4 \mathrm{~B}$ ). This phenomenon might be due to lysis of infected erythrocytes after prolonged in vitro incubation and would mimic the results obtained when DC are incubated with lysates of infected erythrocytes (Figure 1A).

It was also investigated whether the presence of additional uninfected erythrocytes in the cultures of DC with infected erythrocytes, which resembles more closely the situation in vivo, would have an effect on parasitemediated inhibition of DC maturation. When 70 uninfected erythrocytes:DC were added together with 30 infected erythrocytes:DC, mimicking a 30\% parasitaemia infection, a complete inhibition of LPS-induced maturation was observed (Figure 4A, B), suggesting that uninfected erythrocytes do not interfere with the parasite-mediated effect on DC.

It was then assessed whether the intra-erythrocytic stage of parasite development played a role in the inhibition of DC maturation. Each of the parasite stages were added at a similar erythrocyte:DC ratio (30:1). Interestingly, purified $P$. yoelii-infected erythrocytes containing parasites at the trophozoite stage had only a

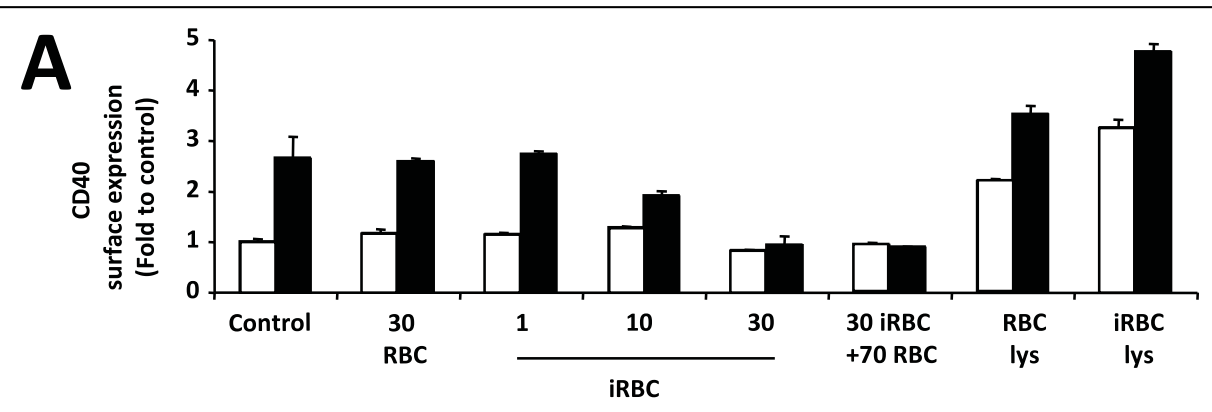

B

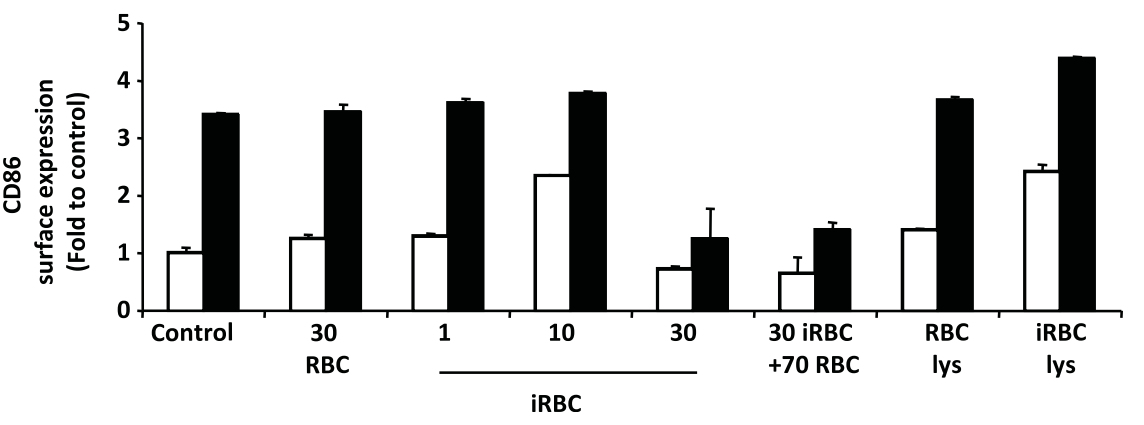

Figure $4 P$. yoelii-infected erythrocytes inhibit DC maturation in response to the TLR4 ligand LPS. Bone-marrow derived DC were incubated for $24 \mathrm{~h}$ in different conditions and then further stimulated with LPS (black bars) or not (white bars) for 18 h. CD86 and CD40 expression determined by flow cytometry are expressed as fold-to-control (DC alone). CD40 (A) and CD86 (B) expression after incubation of DC for $24 \mathrm{~h}$ with RBC or P. yoelii-iRBC at different ratios, as well as RBC or iRBC lysates (lys) and with or without LPS for another $18 \mathrm{~h}$. Data correspond to the average of triplicate samples \pm standard deviation and figures are representative of one out of two independent experiments. 
partial inhibitory effect compared to the inhibition induced by the schizont stage. The immature ring-stage parasites had no effect on inhibition of DC maturation (Figure 5A).

When schizonts were extracted from infected erythrocytes before incubation with $\mathrm{DC}$, thereby removing most erythrocyte components, they also induced complete inhibition of LPS-induced maturation (Figure 5A). This observation suggests that there might be an inhibitory factor that is localized in the parasite itself and not in the host erythrocyte. Indeed, a P. yoelii-derived soluble factor inhibits the maturation of DC [35].

It was also observed that fixation of the infected erythrocytes in the schizont stage did not inhibit LPSinduced DC maturation (Figure 5A). These observations suggest that metabolically active parasites are required for the observed inhibition of DC maturation to take place.

To assess if the inhibitory effect of $P$. yoelii-infected erythrocytes on DC maturation required continuous presence of infected erythrocytes, the infected erythrocytes were rinsed after 3 , or else $24 \mathrm{~h}$ of incubation. Unlike the continuous incubation for $48 \mathrm{~h}$, removal of infected erythrocytes allowed maturation of DC to occur in response to LPS (Figure $5 \mathrm{~B}$ ). These results suggest that the inhibitory effect is reversible and that DC can fully mature upon removal of infected erythrocytes. However, the recovery of maturation response in this experiment may be due to removal of molecules produced by metabolically active parasites that are present in the co-culture supernatant and are responsible for the inhibition of DC maturation, rather than removal of the infected erythrocytes themselves.

\section{Uninfected erythrocytes do not inhibit LPS-induced maturation, even if phagocytosed at a high rate}

Macrophage function is decreased by excessive erythrophagocytosis [36], as well as by phagocytosis of P. falciparum-infected erythrocytes [37]. In light of previous results, it was possible that the excessive phagocytosis of erythrocytes components could be the cause of the absence of DC maturation and resistance to LPS-induced maturation. As Plasmodium-infected erythrocytes are phagocytosed with significantly higher efficiency compared to uninfected erythrocytes [16], control erythrocytes were treated with ionomycin to increase their phagocytosis, since this treatment triggers phosphatidylserine flipping and an apoptosis-like phenotype in

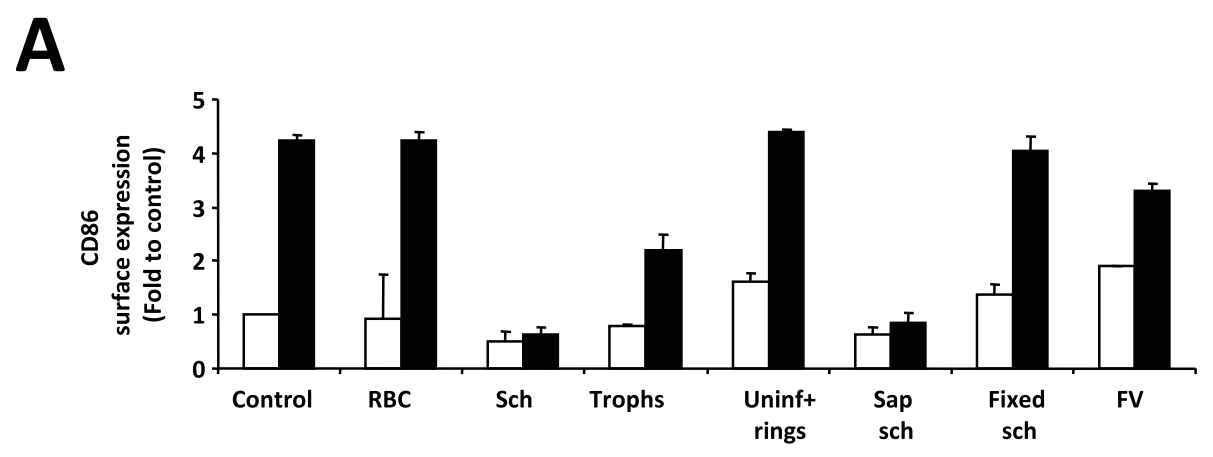

B

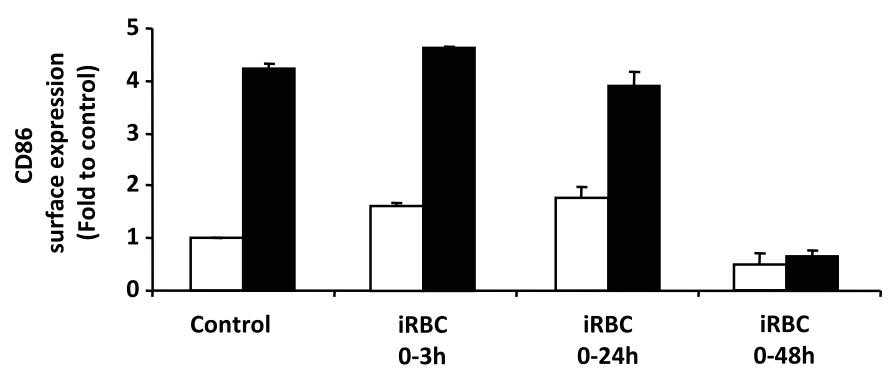

Figure $\mathbf{5}$ The schizont stage of $\boldsymbol{P}$. yoelii-infected erythrocytes inhibit DC maturation in response to LPS. Bone-marrow derived DC were incubated for $24 \mathrm{~h}$ in different conditions and then further stimulated with LPS (black bars) or not (white bars) for $18 \mathrm{~h}$. (A) CD86 expression of DC co-incubated with iRBC at the schizont stage (Sch), the trophozoite stage (Trophs), a mix of uninfected RBC and rings (Uninf+rings), fixed iRBC, saponized iRBC or isolated food vacuoles (FV) for $24 \mathrm{~h}$, followed by addition of LPS and incubation for another $18 \mathrm{~h}$. (B) CD86 expression of DC incubated with iRBC for 0-3, 0-24 or 0-48 h, with addition of LPS at $24 \mathrm{~h}$. Data correspond to the average of triplicate samples \pm standard deviation and figures are representative of one out of two independent experiments. 
erythrocytes [38,39]. Using fluorescently-labeled erythrocytes in these experimental conditions, it was possible to confirm that uninfected control erythrocytes were phagocytosed with low efficiency when compared to infected erythrocytes $(27.7 \%$ and $60.5 \%$ respectively, Figure $6 \mathrm{~A}$, left and middle panels). After treatment of erythrocytes with ionomycin for $30 \mathrm{~min}$, significant flipping of phosphatidyl-serine to the outer membrane was observed by staining with Annexin-V. As expected, erythrocytes treated with ionomycin were phagocytosed very efficiently, to a level comparable to that of $P$. yoelii-infected erythrocytes at the schizont stage (Figure 6A, right panel).

Interestingly, phagocytosis of infected erythrocytes increases with the maturation stage of the parasite, resulting in very efficient phagocytosis of schizonts, intermediate phagocytosis of throphozoites and reduced phagocytosis of ring stages (Figure 6B). This would be compatible with the progressive modifications that are observed in the membrane of infected erythrocytes during an infection cycle [40]. Since there is a good correlation between the level of DC maturation inhibition (Figure 5A) and the level of phagocytosis (Figure 6B) with the different developmental stages, it was determined whether high levels of phagocytosis of uninfected erythrocytes would affect DC maturation. Ionomycintreated erythrocytes did not induce DC maturation (Figure 6C) but failed to prevent LPS-induced maturation (Figure 6D), as observed previously with $P$. yoeliiinfected erythrocytes. To confirm that the most relevant component of erythrocytes does not interfere with DC maturation, DC were preincubated with haemoglobin isolated from control erythrocytes. DC maturation was not affected, even when used at a concentration as high as $1 \mathrm{mg} / \mathrm{ml}$ (Figure 6D). These results exclude the possibility of unspecific effects due to different levels of phagocytosis of infected and uninfected erythrocytes and also confirm the ability of DC to mature even after high levels of erythrophagocytosis.

\section{Kinetics of the inhibition of DC maturation by $P$. yoelii- infected erythrocytes}

The results indicate that $P$. yoelii-infected erythrocytes inhibit the maturation of DC in a reversible manner. The kinetics of this inhibition was analysed by testing different times of pre-incubation of DCs with infected erythrocytes before the addition of LPS as a maturation stimulus. The unresponsiveness of DC increased gradually with the time of pre-incubation with infected erythrocytes, with a maximal inhibitory response obtained after $24 \mathrm{~h}$ of incubation (Figure 7A, B). The expression of IL-12, a cytokine usually secreted upon DC activation, and which is required for induction of Th1 type responses [2], was also assessed. Plasmodium yoeliiinfected erythrocytes inhibited IL-12 secretion and that, interestingly, the pre-incubation time required to obtain maximal inhibition of IL-12 secretion was shorter compared to the inhibition of surface expression of co-stimulatory molecules, which might reflect a posttranscriptional, rather than a transcriptional, regulatory mechanism (Figure 7C).

\section{Plasmodium yoelii-infected erythrocytes inhibit maturation induced by stimulation of different TLRs}

To investigate if the inhibition of DC maturation induced by $P$. yoelii-infected erythrocytes affected stimulation of DC by different TLRs, DC were pre-incubated with infected erythrocytes for $24 \mathrm{~h}$ before stimulation with specific ligands of the following TLRs: TLR1/2 (P3C), TLR2 (zymosan), TLR3 (polyI:C), TLR5 (flagellin), TLR7 (loxoribine), TLR9 (CpG), as well as TLR4 (LPS). For all TLRs studied, the up-regulation of co-stimulatory molecules in DC was severely inhibited by pre-incubation for $24 \mathrm{~h}$ with $P$. yoelii-infected erythrocytes in a dose-dependent fashion (Figure 8). These results suggest that the inhibition of DC maturation induced by P. yoelii-infected erythrocytes is a broad phenomenon that is not restricted to a specific TLR pathway.

\section{Discussion}

Several pathogens are able to induce maturation of DC in vitro in the mouse and human systems. For most of these pathogens, TLR ligands responsible for DC maturation have been isolated, including apicomplexan parasites such as Toxoplasma [25], Trypanosoma [23], and also Plasmodium [26-28]. However, in both Toxoplasma and Plasmodium cases, several reports suggest that DC maturation can be suppressed by whole parasites either in vitro or in vivo [6,41].

In this study, it was shown that erythrocytes infected with the rodent malaria parasite $P$. yoelii have two separate effects on DC maturation in vitro: (1) parasites induce DC maturation only in certain conditions, which seem to depend on the integrity and dose of infected erythrocytes; also (2) there is a threshold above which whole infected erythrocytes inhibit responsiveness of DC to TLR stimulation after continuous incubation for several hours. This dual response may explain apparently controversial results obtained by different laboratories, since Plasmodium-infected erythrocytes from rodent and human species have been found to either activate [12-14], or else inhibit [7-11] DC maturation.

Regarding the activation of DC, it was found that whole $P$. yoelii-infected erythrocytes only induce nonreproducible, weak in vitro DC maturation (one out of two markers) and only in limited conditions. On the other hand, lysates of $P$. yoelii-infected erythrocytes induced up-regulation of CD40, CD80, CD86, MHC-I 


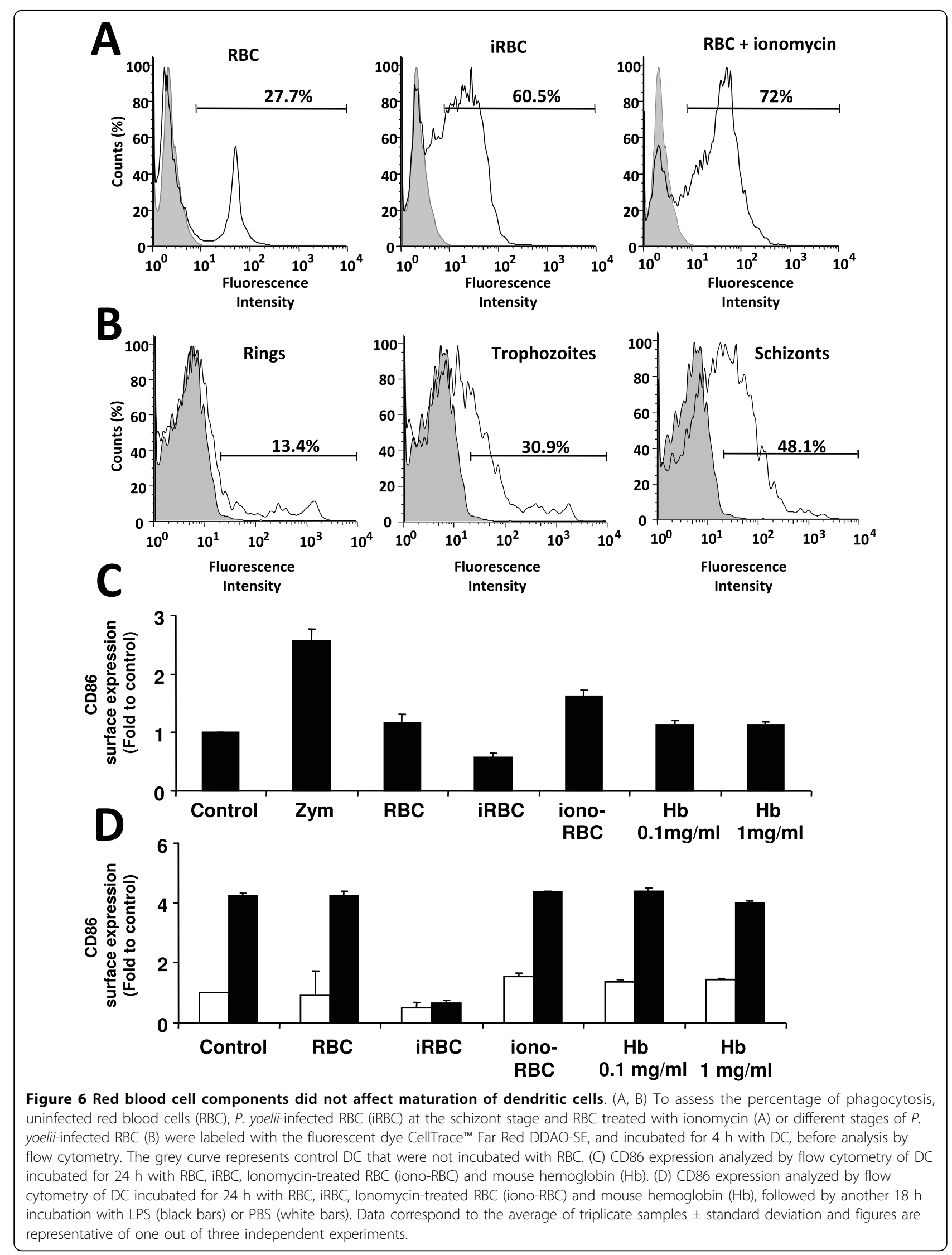



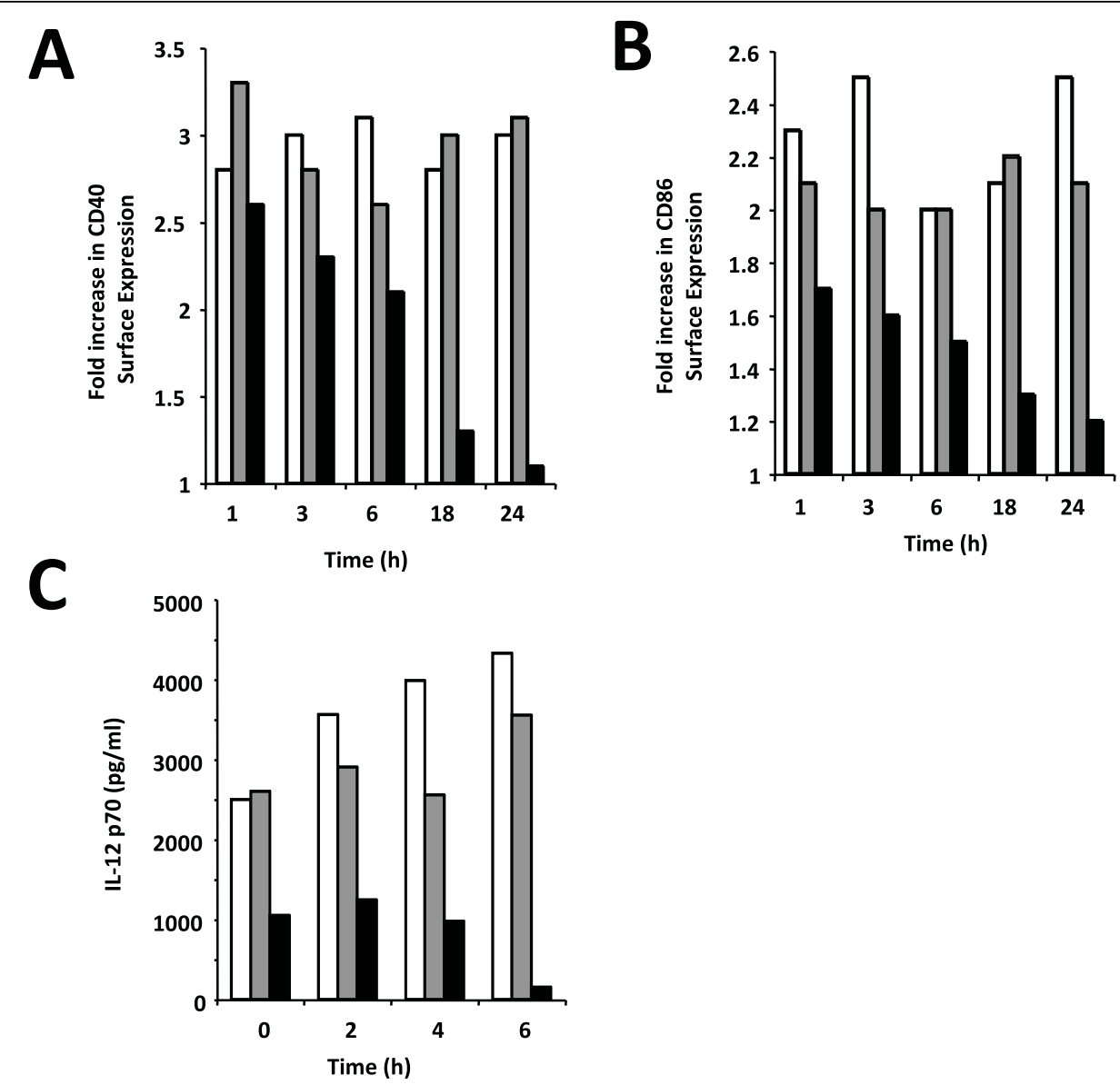

Figure 7 Inhibition of LPS-induced maturation by $P$. yoelii-infected red blood cells (iRBC) is established between 6 and 18 h. To investigate the kinetics of the $\mathrm{RBC}$-induced inhibition of DC maturation, DC were pre-incubated for different times (1 to $24 \mathrm{~h})$ with no RBC (white bars), uninfected RBC at 30:1 (grey bars) or iRBC at 30:1 (black bars), and then pulsed with LPS for 18 h. Cells were then analyzed by flow cytometry for expression of CD40 (A) and CD86 (B). To characterize the kinetics of the iRBC-induced inhibition of cytokine production, DC were pre-incubated for different times (0 to $24 \mathrm{~h}$ ) with uninfected RBC at 30:1 (white bars) or iRBC at 10:1 (grey bars) or 30:1 (black bars), and then pulsed with LPS $1 \mu \mathrm{g} / \mathrm{ml}$. Twenty-four hours after addition of LPS, supernatants of DC cultures were collected and IL-12p70 was quantified by ELISA. Data shows a representative experiment out of two independent experiments.

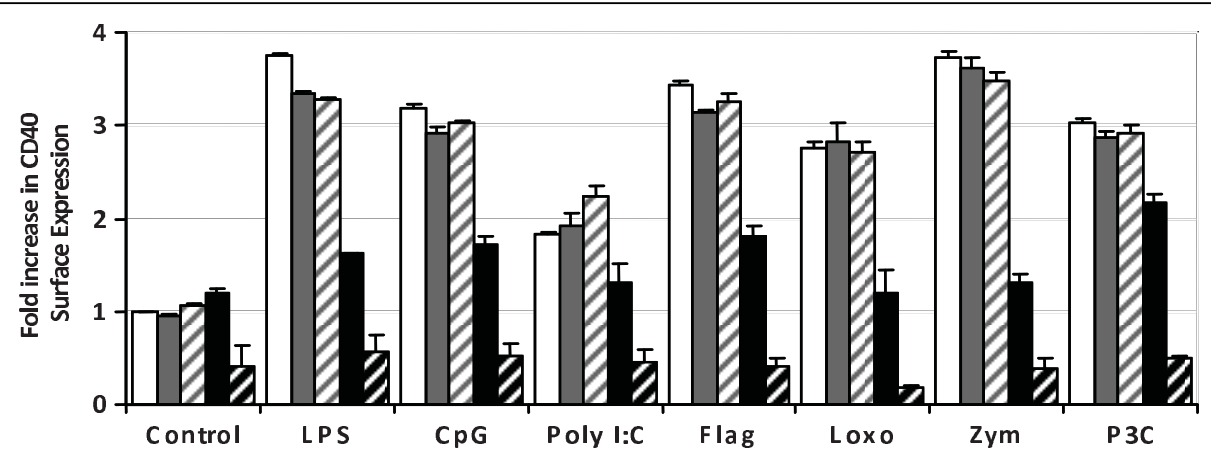

Figure $8 P$. yoelii-infected red blood cells inhibit maturation of dendritic cells induced by stimulation of different Toll-like Receptors. To address the effect of $P$. yoelii-infected RBC (iRBC) on DC maturation induced by activation of different TLRs, DC were pre-incubated for $24 \mathrm{~h}$ with no RBC(white bars), uninfected RBC(gray bars) or iRBC(black bars) at both 10:1(solid bars) and 30:1(striped bars) ratios, and then pulsed with the following ligands of TLRs for an additional 18 hours: LPS $1 \mu \mathrm{g} / \mathrm{ml}$, CpG-ODN $1 \mu \mathrm{g} / \mathrm{ml}$, Zymosan $50 \mu \mathrm{g} / \mathrm{ml}$, Polyl:C $25 \mu \mathrm{g} / \mathrm{ml}$, flagellin $0.1 \mu \mathrm{g} /$ $\mathrm{ml}$ and loxoribin $0.5 \mu \mathrm{M}$. DC were then analyzed by flow cytometry for expression of CD86. Data correspond to the average of triplicate samples \pm standard deviation and figures are representative of one out of three independent experiments. 
and MHC-II. This last finding agrees with previous studies on TLR ligands purified from Plasmodium-infected erythrocytes, such as DNA-covered haemozoin $[27,28]$. However, the finding that DC maturation induced is independent of MyD88 suggests that another Plasmodium-derived activatory molecule, uric acid, may be responsible for the DC maturation observed. The results, therefore, indicate that when the infected erythrocytes lyse before contact with DC, these will undergo maturation. A possible explanation is that Plasmodium TLR ligands only become accessible to their receptors on DC after lysis of the erythrocytes when they are released into the medium. Additionally, their activatory effect might be tampered by the presence of other factors in infected erythrocytes that inhibit TLRinduced signaling at higher parasite doses.

It is also described in this study that infected erythrocytes, when present in high densities, prevent DC from up-regulating co-stimulatory molecules and also from secreting IL-12 in response to TLR stimulation. This phenomenon is observed with whole infected erythrocytes, but not with their lysates. A similar dose-dependent in vitro effect of $P$. falciparum-infected erythrocytes on DC has been reported [8]. The Plasmodium-mediated inhibition of DC maturation is reversible, as responsiveness to TLR ligands is restored if infected erythrocytes are removed, but it is also broad, as expression of co-stimulatory molecules is inhibited in response to all seven TLR ligands tested. Since P. yoelii-infected erythrocytes also inhibit the maturation of DC induced by uric acid crystals [35], it appears that Plasmodium might be affecting a downstream step in the signaling cascade of DC maturation that is common to different activation pathways.

The fact that the mature stages of $P$. yoelii-infected erythrocytes are required for complete inhibition of DC maturation suggests that the inhibitory factor accumulates along with the asexual cycle inside erythrocytes. It appears that haemozoin does not mediate the observed inhibition, since the effect was not observed with purified food vacuoles.

Complete inhibition of DC maturation requires preincubation with $P$. yoelii-infected erythrocytes for at least $18 \mathrm{~h}$. The slow development of a non-responsive state in DC may be related with the time necessary to repress gene expression, but also with a delayed release of the inhibitory factor(s) from infected erythrocytes. Still, this time is significantly shorter than the $48 \mathrm{~h}$ needed to observe partial activation of DC (Figure 4B), suggesting that the lysis of infected erythrocytes occurs only during late times of incubation (between 18 and 48 h) and, therefore, the activatory signal is released only after inhibition of DC has been established The fact that DC maturation is not found at high parasite densities might explain why in rodent models of malaria mature
DC are found early on at low parasitaemia, but not when the load of infection is higher [42].

High levels of phagocytosis of uninfected [43], as well as infected $[16,44]$, erythrocytes is characteristic of Plasmodium blood-stage infections. The study attempted to confirm that the inhibition of DC maturation observed at high ratios of infected erythrocytes is specific of the parasite and not an unspecific effect caused by excessive erythrocyte phagocytosis. Erythrocytes can affect macrophage function [36], including antigen presentation [45], and modify the response to LPS in vivo by impairing host defense [46]. High levels of uninfected erythrocyte phagocytosis - induced by ionomycin - did not result in inhibition of DC maturation. Therefore, the uptake of erythrocytes by itself is not sufficient to inhibit DC maturation. Instead, the inhibition of DC maturation is caused specifically by Plasmodium parasite, as observed when isolated schizonts are added to DC. The observation that fixed infected erythrocytes are not able to inhibit DC maturation suggests that active parasite metabolism is required for such an effect. Recently, the inhibition of DC maturation by a soluble factor derived from Plasmodium infected erythrocytes [35] and by free Plasmodium merozoites has been described [47]. Similarly, there was a strong inhibitory activity mediated by isolated schizonts, the parasite stage preceding the release of free merozoites into circulation.

The data presented here indicate that the effects of Plasmodium blood stages on DC are very different depending on the integrity of infected erythrocytes. The human parasite $P$. falciparum induces a positive modulation or priming of TLR responses, which in turn results in increased inflammatory cytokine responses after subsequent stimulation. This is not observed when DC are incubated with other microbes, which suppress subsequent TLR responses [48]. It is possible that this unique characteristic of Plasmodium is related to the modulation of DC maturation observed here. Even if inflammatory cytokines are produced by DC in response to $P$. yoelii-infected erythrocytes [34], DC do not acquire a mature phenotype. This lack of maturation may account for the possibility of subsequent stimulation of cytokine production that would be suppressed after regular DC maturation.

During a malaria infection, DC probably encounter intact infected erythrocytes, but also lysed erythrocytes after each erythrocytic infection cycle. The two phenomena observed - induction of DC maturation by lysates and inhibition of DC maturation by infected erythrocytes - probably take place during a malaria infection, where high concentrations of infected erythrocytes are reached in organs where parasites accumulate $[49,50]$. Which outcome prevails might be determined by the 
amount of infected erythrocytes that accumulate around DC and also by their integrity. These factors will vary with parasitaemia and also cytoadherence properties, leading to changes in the maturation status of DC throughout infection, as suggested before [42]. Indeed, analysis of the whole myeloid DC population from spleens of malaria-infected mice shows a transcriptomic profile very divergent from typically mature DC [17].

Interesting questions are raised by the dual effect of Plasmodium in DC maturation and its likely role in the orchestration of the immune response to the parasite. Understanding of the mechanism of inhibition of DC maturation induced by the parasite would help understand not only the regulation of gene expression in $\mathrm{DC}$, but also the effect of modulated DCs on regulating $\mathrm{CD}^{+}$and $\mathrm{CD} 8^{+} \mathrm{T}$ cells.

\section{Conclusions}

Plasmodium-infected erythrocytes induce opposite effects on DC: lysed infected erythrocytes induce the maturation of DC, while intact infected erythrocytes induce a broad inhibition of DC maturation by different TLR ligands. These observations explain apparently contradictory findings previously published in this field and also provide an insight into the complexity of the immune response generated by Plasmodium in the host.

\section{List of abbreviations}

(DC): Dendritic cells; (TLR): Toll-like Receptors; (GPI): glycophosphatidyl-inositol; (GM-CSF): Granulocyte/ Macrophage colony-stimulating factor; (iRBC): Infected Red Blood Cell; (LPS): Lipopolysaccharide.

\begin{abstract}
Acknowledgements
Daniel Carapau was funded by Fundação para a Ciência e Tecnologia, Portugal (Grant BD/8851/2002). Esther Bettiol was supported by the Swiss National Science Foundation (PBGEA-115080) and the Swiss Foundation for Grants in Biology and Medicine (PASMA-118678). This work was supported by the American Heart Association Grant-in-Aid 0555742T and Burroughs Wellcome Fund Award for Investigators in Pathogenesis of Infectious Disease to $A R$.

\section{Author details}

'Department of Medical Parasitology, New York University School of Medicine, 341 East 25th street, New York, NY 10010, USA. Institute of Physiological Chemistry, Medical School, MTZ, Dresden University of Technology, Fiedlerstr. 42, 01307 Dresden, Germany.
\end{abstract}

\section{Authors' contributions}

EB and DC carried out the DC maturation experiments, conceived the study and helped write the manuscript, CGR carried out the phagocytosis of infected erythrocytes, COM carried out the kinetics of DC inhibition, AR conceived the study and helped write the manuscript. All authors read and approved the final manuscript.

\section{Competing interests}

The authors declare that they have no competing interests.

Received: 1 April 2009 Accepted: 1 March 2010 Published: 1 March 2010

\section{References}

1. Good MF, Xu H, Wykes M, Engwerda CR: Development and regulation of cell-mediated immune responses to the blood stages of malaria: implications for vaccine research. Annu Rev Immunol 2005, 23:69-99.

2. Guermonprez P, Valladeau J, Zitvogel L, Thery C, Amigorena S: Antigen presentation and T cell stimulation by dendritic cells. Annu Rev Immunol 2002, 20:621-667.

3. Blander JM: Coupling Toll-like receptor signaling with phagocytosis: potentiation of antigen presentation. Trends Immunol 2007, 28:19-25.

4. Kool M, Petrilli V, De Smedt T, Rolaz A, Hammad H, van Nimwegen M, Bergen IM, Castillo R, Lambrecht BN, Tschopp J: Cutting edge: alum adjuvant stimulates inflammatory dendritic cells through activation of the NALP3 inflammasome. J Immunol 2008, 181:3755-3759.

5. Franchi L, Eigenbrod T, Munoz-Planillo R, Nunez G: The inflammasome: a caspase-1-activation platform that regulates immune responses and disease pathogenesis. Nat Immunol 2009, 10:241-247.

6. Wykes MN, Good MF: What really happens to dendritic cells during malaria? Nat Rev Microbiol 2008, 6:864-870.

7. Urban BC, Ferguson DJ, Pain A, Willcox N, Plebanski M, Austyn JM, Roberts DJ: Plasmodium falciparum-infected erythrocytes modulate the maturation of dendritic cells. Nature 1999, 400:73-77.

8. Elliott SR, Spurck TP, Dodin JM, Maier AG, Voss TS, Yosaatmadja F, Payne PD, McFadden Gl, Cowman AF, Rogerson SJ, Shofield L, Brown GV: Inhibition of dendritic cell maturation by malaria is dose dependent and does not require Plasmodium falciparum erythrocyte membrane protein 1. Infect Immun 2007, 75:3621-3632

9. Ocana-Morgner C, Mota MM, Rodriguez A: Malaria blood stage suppression of liver stage immunity by dendritic cells. J Exp Med 2003, 197:143-151.

10. Pouniotis DS, Proudfoot O, Bogdanoska V, Apostolopoulos V, Fifis T, Plebanski M: Dendritic cells induce immunity and long-lasting protection against blood-stage malaria despite an in vitro parasite-induced maturation defect. Infect Immun 2004, 72:5331-5339.

11. Millington OR, Di Lorenzo C, Phillips RS, Garside P, Brewer JM: Suppression of adaptive immunity to heterologous antigens during Plasmodium infection through hemozoin-induced failure of dendritic cell function. J Biol 2006, 5:5.

12. Perry JA, Rush A, Wilson RJ, Olver CS, Avery AC: Dendritic cells from malaria-infected mice are fully functional APC. J Immunol 2004, 172:475-482.

13. Pichyangkul S, Yongvanitchit K, Kum-arb U, Hemmi H, Akira S, Krieg AM, Heppner DG, Stewart VA, Hasegawa H, Looareesuwan S, Shanks GD, Miller RS: Malaria blood stage parasites activate human plasmacytoid dendritic cells and murine dendritic cells through a Toll-like receptor 9-dependent pathway. J Immunol 2004, 172:4926-4933.

14. Seixas E, Cross C, Quin S, Langhorne J: Direct activation of dendritic cells by the malaria parasite, Plasmodium chabaudi chabaudi. Eur J Immunol 2001, 31:2970-2978.

15. Leisewitz AL, Rockett KA, Gumede B, Jones M, Urban B, Kwiatkowski DP: Response of the splenic dendritic cell population to malaria infection. Infect Immun 2004, 72:4233-4239.

16. Ing R, Segura M, Thawani N, Tam M, Stevenson MM: Interaction of mouse dendritic cells and malaria-infected erythrocytes: uptake, maturation, and antigen presentation. J Immunol 2006, 176:441-450.

17. Carapau D, Kruhofer M, Chatalbash A, Orengo JM, Mota MM, Rodriguez A: Transcriptome profile of dendritic cells during malaria: CAMP regulation of IL-6. Cell Microbiol 2007, 9:1738-1752.

18. Millington OR, Gibson VB, Rush CM, Zinselmeyer BH, Phillips RS, Garside P, Brewer JM: Malaria impairs T cell clustering and immune priming despite normal signal 1 from dendritic cells. PLoS Pathog 2007, 3:1380-1387.

19. Urban BC, Cordery D, Shafi MJ, Bull PC, Newbold Cl, Williams TN, Marsh K: The frequency of BDCA3-positive dendritic cells is increased in the peripheral circulation of Kenyan children with severe malaria. Infect Immun 2006, 74:6700-6706.

20. Wykes MN, Liu XQ, Beattie L, Stanisic DI, Stacey KJ, Smyth MJ, Thomas R, Good MF: Plasmodium strain determines dendritic cell function essential for survival from malaria. PLoS Pathog 2007, 3:e96.

21. Wong KA, Rodriguez A: Plasmodium infection and endotoxic shock induce the expansion of regulatory dendritic cells. J Immunol 2008, 180:716-726. 
22. Chen K, Huang J, Gong W, Iribarren P, Dunlop NM, Wang JM: Toll-like receptors in inflammation, infection and cancer. Int Immunopharmacol 2007, 7:1271-1285.

23. Campos MA, Almeida IC, Takeuchi O, Akira S, Valente EP, Procopio DO, Travassos LR, Smith JA, Golenbock DT, Gazzinelli RT: Activation of Toll-like receptor-2 by glycosylphosphatidylinositol anchors from a protozoan parasite. J Immunol 2001, 167:416-423.

24. Becker I, Salaiza N, Aguirre M, Delgado J, Carrillo-Carrasco N, Kobeh LG, Ruiz A, Cervantes R, Torres AP, Cabrera N, Gonzalez A, Maldonado C, Isibasi A: Leishmania lipophosphoglycan (LPG) activates NK cells through toll-like receptor-2. Mol Biochem Parasitol 2003, 130:65-74.

25. Yarovinsky F, Zhang D, Andersen JF, Bannenberg GL, Serhan CN, Hayden MS, Hieny S, Sutterwala FS, Flavell RA, Ghosh S, Sher A: TLR11 activation of dendritic cells by a protozoan profilin-like protein. Science 2005, 308:1626-1629.

26. Krishnegowda G, Hajjar AM, Zhu J, Douglass EJ, Uematsu S, Akira S, Woods AS, Gowda DC: Induction of proinflammatory responses in macrophages by the glycosylphosphatidylinositols of Plasmodium falciparum: cell signaling receptors, glycosylphosphatidylinositol (GPI) structural requirement, and regulation of GPI activity. J Biol Chem 2005, 280:8606-8616

27. Coban C, Ishii KJ, Kawai T, Hemmi H, Sato S, Uematsu S, Yamamoto M, Takeuchi O, Itagaki S, Kumar N, HOrii T, Akira S: Toll-like receptor 9 mediates innate immune activation by the malaria pigment hemozoin. J Exp Med 2005, 201:19-25.

28. Parroche P, Lauw FN, Goutagny N, Latz E, Monks BG, Visintin A, Halmen KA, Lamphier M, Olivier M, Bartholomeu DC, Gazzinelli RT, Golenbock DT: Malaria hemozoin is immunologically inert but radically enhances innate responses by presenting malaria DNA to Toll-like receptor 9. Proc Natl Acad Sci USA 2007, 104:1919-1924.

29. Bruna-Romero O, Rodriguez A: Dendritic cells can initiate protective immune responses against malaria. Infect Immun 2001, 69:5173-5176.

30. Kariuki MM, Kiaira JK, Mulaa FK, Mwangi JK, Wasunna MK, Martin SK: Plasmodium falciparum: purification of the various gametocyte developmental stages from in vitro-cultivated parasites. Am J Trop Med Hyg 1998, 59:505-508.

31. Saliba KJ, Folb PI, Smith PJ: Role for the Plasmodium falciparum digestive vacuole in chloroquine resistance. Biochem Pharmacol 1998, 56:313-320.

32. Schofield L, Hackett F: Signal transduction in host cells by a glycosylphosphatidylinositol toxin of malaria parasites. J Exp Med 1993, 177:145-153.

33. Orengo JM, Evans JE, Bettiol E, Leliwa-Sytek A, Day K, Rodriguez A: Plasmodium-induced inflammation by uric acid. PLoS Pathog 2008, 4 : e1000013.

34. Orengo JM, Leliwa-Sytek A, Evans JE, Evans B, Hoef van de D, Nyako M, Day K, Rodriguez A: Uric acid is a mediator of the Plasmodium falciparum-induced inflammatory response. PLoS One 2009, 4:e5194.

35. Orengo JM, Wong KA, Ocana-Morgner C, Rodriguez A: A Plasmodium yoelii soluble factor inhibits the phenotypic maturation of dendritic cells. Malar J 2008, 7:254.

36. Commins LM, Loegering DJ, Gudewicz PW: Effect of phagocytosis of erythrocytes and erythrocyte ghosts on macrophage phagocytic function and hydrogen peroxide production. Inflammation 1990, 14:705-716.

37. Schwarzer E, Turrini F, Ulliers D, Giribaldi G, Ginsburg $H$, Arese $P$ : Impairment of macrophage functions after ingestion of Plasmodium falciparum-infected erythrocytes or isolated malarial pigment. J Exp Med 1992, 176:1033-1041

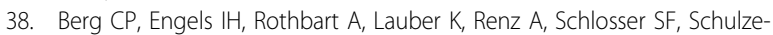
Osthoff $K$, Wesselborg $S$ : Human mature red blood cells express caspase3 and caspase- 8 , but are devoid of mitochondrial regulators of apoptosis. Cell Death Differ 2001, 8:1197-1206.

39. Bratosin D, Estaquier J, Petit F, Arnoult D, Quatannens B, Tissier JP, Slomianny C, Sartiaux C, Alonso C, Huart JJ, Montreuil J, Ameisen JC: Programmed cell death in mature erythrocytes: a model for investigating death effector pathways operating in the absence of mitochondria. Cell Death Differ 2001, 8:1143-1156.

40. Maier AG, Cooke BM, Cowman AF, Tilley L: Malaria parasite proteins that remodel the host erythrocyte. Nat Rev Microbiol 2009, 7:341-354.
41. McKee AS, Dzierszinski F, Boes M, Roos DS, Pearce EJ: Functional inactivation of immature dendritic cells by the intracellular parasite Toxoplasma gondii. J Immunol 2004, 173:2632-2640.

42. Perry JA, Olver CS, Burnett RC, Avery AC: Cutting edge: the acquisition of TLR tolerance during malaria infection impacts $T$ cell activation. $\mathrm{J}$ Immunol 2005, 174:5921-5925.

43. Salmon MG, De Souza JB, Butcher GA, Playfair JH: Premature removal of uninfected erythrocytes during malarial infection of normal and immunodeficient mice. Clin Exp Immunol 1997, 108:471-476.

44. Bettiol E, Hoef van de DL, Carapau D, Rodriguez A: Efficient phagosomal maturation and degradation of Plasmodium-infected erythrocytes by dendritic cells and macrophages. Parasite Immunology .

45. Carrasco-Marin E, Alvarez-Dominguez C, Lopez-Mato P, Martinez-Palencia R, Leyva-Cobian F: Iron salts and iron-containing porphyrins block presentation of protein antigens by macrophages to MHC class II-restricted T cells. Cell Immunol 1996, 171:173-185.

46. Loegering DJ, Commins LM, Minnear FL, Gary LA, Hill LA: Effect of Kupffer cell phagocytosis of erythrocytes and erythrocyte ghosts on susceptibility to endotoxemia and bacteremia. Infect Immun 1987, 55:2074-2080.

47. Mukherjee $P$, Chauhan VS: Plasmodium falciparum-free merozoites and infected RBCs distinctly affect soluble CD40 ligand-mediated maturation of immature monocyte-derived dendritic cells. J Leukoc Biol 2008, 84:244-254.

48. McCall MB, Netea MG, Hermsen CC, Jansen T, Jacobs L, Golenbock D, Ven van der AJ, Sauerwein RW: Plasmodium falciparum infection causes proinflammatory priming of human TLR responses. J Immunol 2007, 179:162-171.

49. Franke-Fayard B, Janse CJ, Cunha-Rodrigues M, Ramesar J, Buscher P, Que I, Lowik C, Voshol PJ, den Boer MA, van Duinen SG, Febbraio M, Mota MM, Waters AP: Murine malaria parasite sequestration: CD36 is the major receptor, but cerebral pathology is unlinked to sequestration. Proc Natl Acad Sci USA 2005, 102:11468-11473.

50. Mota MM, Jarra W, Hirst E, Patnaik PK, Holder AA: Plasmodium chabaudiinfected erythrocytes adhere to CD36 and bind to microvascular endothelial cells in an organ-specific way. Infect Immun 2000, 68:4135-4144

doi:10.1186/1475-2875-9-64

Cite this article as: Bettiol et al:: Dual effect of Plasmodium-infected erythrocytes on dendritic cell maturation. Malaria Journal 2010 9:64.

\section{Submit your next manuscript to BioMed Central and take full advantage of:}

- Convenient online submission

- Thorough peer review

- No space constraints or color figure charges

- Immediate publication on acceptance

- Inclusion in PubMed, CAS, Scopus and Google Scholar

- Research which is freely available for redistribution

Submit your manuscript at www.biomedcentral.com/submit
Biomed Central 